\title{
Central venous catheter-related bloodstream infections in patients with hematological malignancies: Comparison of data from a clinical registry and a randomized controlled trial
}

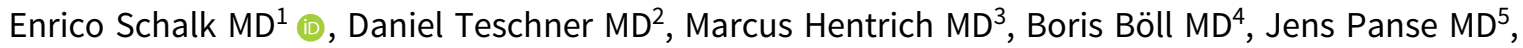 \\ Martin Schmidt-Hieber $\mathrm{MD}^{6}$, Maria J.G.T. Vehreschild MD ${ }^{4,7,8}$ and Lena M. Biehl MD ${ }^{4,7}$ \\ ${ }^{1}$ Department of Hematology and Oncology, Otto-von-Guericke University Magdeburg, Medical Center, Magdeburg, Germany, ${ }^{2}$ Department of Hematology, Medical \\ Oncology, and Pneumology, University Medical Center of the Johannes Gutenberg University Mainz, Mainz, Germany, ${ }^{3}$ Department of Hematology and Oncology, \\ Red Cross Hospital Munich, Munich, Germany, ${ }^{4}$ Department I of Internal Medicine, Center for Integrated Oncology Aachen Bonn Cologne Dusseldorf, University of \\ Cologne, Cologne, Germany, ${ }^{5}$ Department of Oncology, Hematology, Hemostaseology and Stem Cell Transplantation, University Hospital RWTH Aachen, Medical \\ Faculty, Aachen, Germany, ${ }^{6} \mathrm{Clinic}$ of Hematology and Oncology, Carl-Thiem Hospital Cottbus, Cottbus, Germany, ${ }^{7}$ German Center for Infection Research (DZIF), \\ Site Bonn/Cologne, Cologne, Germany and ${ }^{8}$ Department of Internal Medicine, Infectious Diseases, Goethe University Frankfurt, Frankfurt am Main, Germany
}

To the Editor-Central venous catheter (CVC)-related bloodstream infections (CRBSIs) are clinically important healthcareassociated infections. ${ }^{1,2}$ To reduce the burden of CRBSIs, many countries have implemented national surveillance programs. ${ }^{3,4}$ Reliable epidemiological data are provided not only by cohort studies but also by randomized controlled trials (RCTs), ${ }^{5-7}$ which are considered the gold standard for assessment of clinically relevant questions. ${ }^{8}$ However, patient populations included in RCTs are often highly selected, limiting the generalizability of study results. In contrast, clinical registries can provide useful data on rare conditions in the daily routine that may also be helpful in assessing quality of care. On the other hand, they are limited by substantial confounders and bias. ${ }^{8}$

Our aim was to compare epidemiological data on CRBSIs in patients with hematological malignancies derived from a RCT (COAT, ${ }^{6}$ ClinicalTrials.gov, no. NCT01544686) with data from a prospective registry (SECRECY, ${ }^{9}$ German Clinical Trial Register, no. DRKS00006551). We hypothesized that patients included in a CVC RCT profit from improved CVC care compared to patients under standard clinical care conditions, possibly resulting in different CRBSI rates. Thus, the primary aim of the present analysis was to compare the CRBSI incidences between these groups.

The COAT trial was a RCT carried out from February 2012 to September 2014 in 10 hematology/oncology departments in Germany to compare 2 different types of CVC dressings with respect to the associated CRBSI incidence in neutropenic patients. ${ }^{6}$ The SECRECY registry began in March 2013 and is an ongoing CRBSI registry, ${ }^{9}$ now in 6 hematology/oncology departments in Germany ${ }^{10}$ In the present analysis, we included data from patients with nontunneled jugular and subclavian vein CVCs placed $\geq 1$ day in situ. For CRBSI diagnosis, we used the 2012 definitions of the Infectious Diseases Working Party (AGIHO) of the German Society of Hematology and Medical Oncology (DGHO), considering only definite or probable CRBSIs. ${ }^{7}$ Because the end points in the COAT trial referred to the $2008 \mathrm{AGIHO} / \mathrm{DGHO}$ definitions, ${ }^{11}$ the underlying data were reassessed, and the more recent definitions were applied to ensure the comparability of the 2 data sets.

\footnotetext{
Author for correspondence: Enrico Schalk, Email: enrico.schalk@med.ovgu.de Cite this article: Schalk E, et al. (2020). Central venous catheter-related bloodstream infections in patients with hematological malignancies: Comparison of data from a clinical registry and a randomized controlled trial. Infection Control \& Hospital Epidemiology, 41: 254-256, https://doi.org/10.1017/ice.2019.335

According to the aforementioned inclusion criteria, we found 610 cases in the completed COAT trial data set and, as of May $2019,2,390$ cases in the SECRECY registry. In this entire cohort of 3,000 CVCs with 49,542 CVC days, underlying diseases were hematological malignancies and solid tumors in $2,719(90.6 \%)$ and $158(5.3 \%)$ cases, respectively. For a matched-pair analysis, the 610 patients from the COAT trial were matched with those patients from the SECRECY registry with regard to the use of chlorhexidine-coated CVC dressings, sex, and underlying disease with a high risk for CRBSI (ie, acute myeloid leukemia [AML], multiple myeloma, or non-Hodgkin lymphoma ${ }^{10}$ ).

Comparison of continuous variables was conducted using the Mann-Whitney $\mathrm{U}$ test, and categorical variables were compared using the Pearson $\chi^{2}$ test or the Fisher exact test, when possible. CRBSI incidences were compared by $\chi^{2}$ test. The Kaplan-Meier method was used for calculating the CRBSI probability over time, and the comparison between both groups was performed using the log-rank test and Cox regression. Two-sided $P$ values $<.05$ were considered statistically significant. Statistical analyses were carried out using SPSS version 24 statistical software (IBM, Armonk, NY).

Patient and CVC characteristics as well as CRBSI features are shown in Table 1. Patients from the COAT cohort were younger than those from the SECRECY cohort (median, 58 vs 60 years; $P<.001$ ). There was a slight male predominance in both groups. COAT included fewer cases of AML than SECRECY (243 of 610 [39.8\%] vs 300 of 610 [49.2\%]; $P=.001$ ) but more cases of multiple myeloma (170 of 610 [27.9\%] vs 123 of 610 [20.2\%]; $P=.002$ ). In the COAT cohort, significantly fewer CVCs were inserted into the jugular vein, compared to the matched SECRECY cohort $(41.8 \%$ vs $94.3 \%$; $P<.001)$. Although the median duration of CVC placement was significantly longer in the COAT trial than in the SECRECY registry ( 18 vs 15 days; $P<.001$ ), the median time to CRBSI onset was significantly shorter in the COAT cohort (12 vs 14 days; $P=.040$ ). We found no difference in the CRBSI rate between the COAT group and the SECRECY group (79 of 610 [13.0\%] vs 71 of 610 [11.6\%]; $P=.542$ ). Furthermore, the proportion of definite and probable CRBSIs was similar in both cohorts $(P=.744)$. Regarding the primary aim of this analysis, the incidence of CRBSIs did not differ significantly with 7.0 CRBSIs per 1,000 CVC days in the COAT trial and 7.3 CRBSIs per 1,000 CVC days in the SECRECY registry $(P=.794)$. Analyzing the CRBSI probability over time (ie, 
Table 1. Patient/CVC Characteristics and CRBSI Features

\begin{tabular}{|c|c|c|c|c|}
\hline Parameter & Whole Cohort $(n=3,000)$ & COAT Trial $(n=610)$ & SECRECY Registry $(n=610)$ & $P$ Value $^{\mathrm{a}}$ \\
\hline Median age, years (IQR) & $59(48-66)$ & $58(48-66)$ & $60(53-68)$ & $<.001^{\mathrm{b}}$ \\
\hline Males, n/N (\%) & $1,759 / 3,000(58.6)$ & $361 / 610(59.2)$ & $361 / 610(59.2)$ & $1.000^{c}$ \\
\hline Underlying disease, $\mathrm{n} / \mathrm{N}(\%)$ & & & & $<.001^{d}$ \\
\hline Acute myeloid leukemia & $1,194 / 3,000(39.8)$ & $243 / 610(39.8)$ & $300 / 610(49.2)$ & \\
\hline Multiple myeloma & $658 / 3,000(21.9)$ & $170 / 610(27.9)$ & $123 / 610(20.2)$ & \\
\hline Non-Hodgkin lymphoma & $529 / 3,000(17.6)$ & $115 / 610(18.9)$ & $105 / 610(17.2)$ & \\
\hline Acute lymphoblastic leukemia & $271 / 3,000(9.1)$ & $34 / 610(5.6)$ & $12 / 610(3.1)$ & \\
\hline Hodgkin lymphoma & $86 / 3,000(2.9)$ & $24 / 610(3.9)$ & $6 / 610(1.0)$ & \\
\hline Other & $316 / 3,000(10.5)$ & $24 / 610(3.9)$ & $57 / 610(9.3)$ & \\
\hline High-risk disease, $\mathrm{n} / \mathrm{N}(\%)^{\mathrm{e}}$ & $2,381 / 3,000(79.4)$ & $528 / 610(86.6)$ & $528 / 610(86.6)$ & $1.000^{c}$ \\
\hline Chlorhexidine-coated CVC dressing, n/N (\%) & $1,271 / 3,000(42.4)$ & $306 / 610(50.2)$ & $306 / 610(50.2)$ & $1.000^{c}$ \\
\hline Jugular vein CVC, n/N (\%) & $2,509 / 3,000(83.6)$ & $255 / 610(41.8)$ & $575 / 610(94.3)$ & $<.001^{\mathrm{c}}$ \\
\hline CVC days, total & 49,542 & 11,221 & 9,664 & \\
\hline CVC days, median (IQR) & $16(9-23)$ & $18(14-24)$ & $15(7-23)$ & $<.001^{f}$ \\
\hline Median time to CRBSI onset, d (IQR) & $14(11-20)$ & $12(10-16)$ & $14(11-20)$ & $.040^{\mathrm{b}}$ \\
\hline CRBSI, n/N (\%) & $335 / 3,000(11.2)$ & $79 / 610(13.0)$ & $71 / 610(11.6)$ & $.542^{\mathrm{c}}$ \\
\hline Definitive, n/N (\%) & $175 / 335(52.2)$ & $37 / 79(46.8)$ & $36 / 71(50.7)$ & $.744^{c}$ \\
\hline Probable, $\mathrm{n} / \mathrm{N}(\%)$ & $160 / 335(47.8)$ & $42 / 79(53.2)$ & $35 / 71(49.3)$ & \\
\hline CRBSI incidence, $x / 1,000$ CVC days & 6.8 & 7.0 & 7.3 & $.794^{\mathrm{g}}$ \\
\hline CRBSI14, \% (95\% Cl) & $8.5(7.3-9.7)$ & $10.4(7.7-13.1)$ & $9.6(6.7-12.5)$ & $.580^{f}$ \\
\hline Causative pathogens, n/N (\%) & & & & $.142^{\mathrm{d}}$ \\
\hline Coagulase-negative staphylococci & $241 / 335(71.9)$ & $49 / 79(62.0)$ & $51 / 71(71.8)$ & \\
\hline Other Gram-positive bacteria & $34 / 335(10.1)$ & $10 / 79(12.7)$ & $3 / 71(4.2)$ & \\
\hline Enterobacteriaceae & $20 / 335(6.0)$ & $8 / 79(10.1)$ & $4 / 71(5.6)$ & \\
\hline Other Gram-negative bacteria & $12 / 335(6.6)$ & $2 / 79(2.5)$ & $6 / 71(8.5)$ & \\
\hline Candida spp & $10 / 335(3.0)$ & $2 / 79(2.5)$ & $3 / 71(4.2)$ & \\
\hline Multibacterial & $18 / 335(5.4)$ & $8 / 79(10.1)$ & $4 / 71(5.6)$ & \\
\hline
\end{tabular}

Note. CVC, central venous catheter; CRBSI, central venous catheter-related bloodstream infection; IQR, interquartile range; CRBSI14, cumulative CRBSI probability at day 14; $95 \% \mathrm{Cl}, 95 \%$ confidence interval.

${ }^{a}$ Comparison COAT trial data vs matched-pair data from SECRECY registry; all $P$ values are 2-sided.

bMann-Whitney U test.

'Fisher's exact test.

'Pearson's $\chi^{2}$ test.

eUnderlying disease with high risk for CRBSI: acute myeloid leukemia, multiple myeloma or non-Hodgkin lymphoma. ${ }^{10}$

flog-rank test.

$\mathrm{g}^{2}$ test.

cumulative CRBSI incidence), we also found no significant differences at day 14 between these groups $(10.4 \%$ in the COAT trail vs $9.6 \%$ in the SECRECY registry: hazard ratio, 1.12 ; $95 \%$ confidence interval, $0.74-1.71 ; P=.583)$. Finally, we detected no differences in the distribution of pathogen subgroups $(P=.142)$, and coagulase-negative staphylococci were the most common causative pathogens for CRBSIs in both groups.

To the best of our knowledge, the present analysis is the first to provide comparative epidemiological data on CRBSIs from a RCT and a registry study in high-risk patients with hematological malignancies. Although limited by some differences in patient and CVC characteristics between both groups, this large matched-pair analysis did not show a reduced CRBSI incidence in patients treated in a RCT compared to patients in routine clinical care implicating a comparable quality of CVC care. Thus, epidemiological data on
CRBSIs derived from RCTs may reliably be transferred to realworld settings.

Acknowledgments. The authors thank all COAT investigators involved in the conduct of the trial and all SECRECY investigators for their great support and documentation.

Financial support. No financial support was provided relevant to this article.

Conflicts of interest. D.T. reports grants and personal fees from Gilead, Pfizer, and MSD, grants from Abbvie, Astellas, Celgene, and Jazz outside this work. M.H. has served on advisory boards of Amgen, BMS, Hexal, Janssen, Jazz Pharma, Roche, Sanofi, and Takeda; has served on the speakers' bureaus of Amgen, BMS, Janssen, Sanofi, and Takeda; and has received travel grants from Amgen, Janssen, and Takeda. B.B. has served at the speaker's bureau of Astellas, Celgene, Johnson \& Johnson, Kite/Gilead, MSD, Novartis, and Takeda; has received research funding from Astellas, Kite/Gilead and MSD; and has 
consulted for Kite/Gilead, MSD, Novartis, and Takeda. J.P. reports personal fees from Pfizer, Alexion, Apellis, BMS, Celgene, Novartis, Hexal AG, Boehringer Ingelheim, and Chugai Pharma outside the submitted work. M.S.H. reports financial support of educational meetings at the Carl-Thiem-Klinikum Cottbus, Germany, by Janssen-Cilag GmbH, Takeda Pharma Vertrieb GmbH \& Co. KG, Novartis Pharma Oncology, Pfizer Pharma GmbH, Roche Pharma AG, Vifor Pharma Deutschland GmbH, and Celgene GmbH. M.J.G.T.V. has served at the speakers' bureau of Akademie für Infektionsmedizin, Ärztekammer Nordrhein, Astellas Pharma, Basilea, Gilead Sciences, Merck/MSD, Organobalance, and Pfizer; has received research funding from 3M, Evoinik, Glycom, Astellas Pharma, DaVolterra, Gilead Sciences, MaaT Pharma, Merck/MSD, Morphochem, Organobalance, and Seres Therapeutics; and is a consultant to Alb-Fils Kliniken GmbH, Arderypharm, Astellas Pharma, Ferring, DaVolterra, MaaT Pharma, and Merck/MSD. L.M.B. reports lecture honoraria from Astellas and MSD and travel grants from 3M and Gilead. E.S. reports no conflicts of interest relevant to this article.

\section{References}

1. Magill SS, O'Leary E, Janelle SJ, et al. Changes in prevalence of healthcareassociated infections in US hospitals. N Engl J Med 2018;379:1732-1744.

2. Battaglia CC, Hale K. Hospital-acquired infections in critically ill patients with cancer. J Intensive Care Med 2019;34:523-536.

3. Hallam C, Jackson T, Rajgopal A, Russell B. Establishing catheter-related bloodstream infection surveillance to drive improvement. J Infect Prev 2018;19:160-166.

4. Surveillance of nosocomial infections as well as the detection of pathogens with special resistance and multi-resistance [in German]. Bundesgesundheitsblatt Gesundheitsforschung Gesundheitsschutz 2013;56:580-583.
5. Parienti JJ, Mongardon N, Mégarbane B, et al. Intravascular complications of central venous catheterization by insertion site. N Engl J Med 2015; 373:1220-1229.

6. Biehl LM, Huth A, Panse J, et al. A randomized trial on chlorhexidine dressings for the prevention of catheter-related bloodstream infections in neutropenic patients. Ann Oncol 2016;27:1916-1922.

7. Hentrich M, Schalk E, Schmidt-Hieber M, et al. Central venous catheterrelated infections in hematology and oncology: 2012 updated guidelines on diagnosis, management and prevention by the Infectious Diseases Working Party of the German Society of Hematology and Medical Oncology. Ann Oncol 2014;25:936-947.

8. Frieden TR. Evidence for health decision making-beyond randomized, controlled trials. N Engl J Med 2017;377:465-475.

9. Schalk E, Hanus L, Färber J, Fischer T, Heidel FH. Prediction of central venous catheter-related bloodstream infections (CRBSIs) in patients with haematologic malignancies using a modified Infection Probability Score (mIPS). Ann Hematol 2015;94:1451-1456.

10. Schalk E, Toelle D, Schulz S, et al. Identifying haematological cancer patients with high risk for central venous catheter (CVC)-related bloodstream infections at the time point of CVC insertion (abstract P2556). Presented at the $29^{\text {th }}$ European Congress of Clinical Microbiology and Infectious Diseases, April 13-16, 2019, Amsterdam, The Netherlands.

11. Wolf HH, Leithauser M, Maschmeyer G, et al. Central venous catheterrelated infections in hematology and oncology: guidelines of the Infectious Diseases Working Party (AGIHO) of the German Society of Hematology and Oncology (DGHO). Ann Hematol 2008;87:863-876.

\title{
A pseudo-outbreak of Legionnaires' disease in an acute-care hospital
}

\author{
Roland Schulze-Röbbecke (1) \\ Department of Infection Control and Infectious Diseases, RWTH University Hospital Aachen, Aachen, Germany
}

To the Editor-Epidemics of Legionnaires' disease (legionellosis) may involve large numbers of cases, with case-fatality rates of about $10 \%$ overall, and $25 \%$ in healthcare-associated cases. ${ }^{1}$ The sources of legionellosis outbreaks are usually building water systems and devices, including potable water and cooling towers; therefore, any suspicion of a legionellosis outbreak must prompt efforts to identify the source and to stop further transmission.

This is a report on a suspected healthcare-associated legionellosis outbreak in an acute-care hospital involving 10 inpatients who tested positive for urinary antigen of Legionella pneumophila serogroup 1 between January and April 2017. Of 160 urinary antigen tests performed during this time period, 10 yielded positive results (positivity rate, 6.3\%). In the previous year, only 1 of 76 tests had yielded a positive result (positivity rate, 1.3\%).

The public health authority was notified and extensive testing of water samples for Legionellae was ordered. These tests only yielded L. pneumophila of a serogroup other than 1. Patient data showed that 5 patients had signs of pneumonia on admission:

Author for correspondence: Roland Schulze-Röbbecke, PhD, Department of Infection Control and Infectious Diseases, RWTH University Hospital Aachen, Pauwelsstraße 30, 52074 Aachen, Germany. Email: roland.schulze-roebbecke@rwth-aachen.de

Cite this article: Schulze-Röbbecke R. (2020). A pseudo-outbreak of Legionnaires' disease in an acute-care hospital. Infection Control \& Hospital Epidemiology, 41: 256-257, https://doi.org/10.1017/ice.2019.365
1 patient had nonrespiratory signs consistent with legionellosis on admission, and 4 patients did not present any signs of pneumonia during their hospital stay. At the time of intervention, all but 1 patient had been discharged. A urine sample of the last patient was divided into 2 portions. One portion was sent to laboratory A, which had issued the positive test results, and the other portion was sent to laboratory $\mathrm{B}$. In laboratory $\mathrm{A}$, the urine sample again tested positive, whereas in laboratory $\mathrm{B}$, the sample tested negative. Confronted with these findings, laboratory A reported having switched to a new urinary antigen test early in 2017, which later turned out to be of poor specificity. After a healthcareassociated outbreak had been ruled out, public health officials ruled out an outbreak altogether, either because legionellosis was not confirmed in the patients presenting signs of pneumonia or because no epidemiological link was found.

In conclusion, the presumptive healthcare-associated legionellosis outbreak caused considerable unrest within the hospital and among the public health authorities, but it proved to be a pseudo-outbreak. Pseudo-outbreaks (or pseudo-epidemics) are real clusters of false infections or artifactual clusters of real infections. ${ }^{2}$ The pseudo-outbreak described here was caused by false-positive urinary-antigen test results. Community pseudooutbreaks of this kind have been reported previously. ${ }^{3,4}$ In our case, the pseudo-outbreak was complicated by assuming healthcare 